\title{
Research on selective disassembly sequence planning for Repair DENG Mingxing ${ }^{1}$, WANG Jumei ${ }^{1}$, Tang Qiuhua ${ }^{1}$, ZHANG Liping ${ }^{1}$
}

\author{
${ }^{1}$ Wuhan University of Science and Technology, Wuhan 430081, China \\ ycdmx@126.com,tangqiuhua@wust.edu.cn,zhangliping@wust.edu.cn
}

\begin{abstract}
Key words: Selective disassembly; Disassembly Sequence Planning for repair; hybrid precedence graph; stability; ant colony algorithm

Abstract: In order to improve the efficiency of selective disassembly sequence planning for repair, a selective disassembly sequence planning approach based on ant colony algorithm is proposed. Hybrid Precedence Graph(HPG) is constructed to describe the geometric constraints among constituting components and components' stability during disassembly. Then in order to find the target component accurately and quickly, disassembly set for the target is obtained based on HPG. Dynamical candidates set strategy based on precedence relations is adopted to constrain the searching space, and a selective Disassembly Sequence Planning(DSP) approach based on HPG is proposed. Finally, the validity and feasibility of the proposed approach is verified by a study case. Taking geometric constraint and components' stability into count, this approach could be more practical.
\end{abstract}

\section{Introduction}

It is well known that disassembly is the first step for repair, and efficiency of disassembly sequence affects the cost of repair. This procedure usually requires disassembly of nonfunctional components and may involve disassembly of functional components due to disassembly precedence relationships. Disassembly for repair always means selective disassembly, and the disassembly sequence affects cost of repair. Disassembly sequence planning refers to a task that aims at finding the feasible and optimal disassembly sequence, considering all constraints in the disassembly. In general, the constraints include the precedence relations, geometrical constraint and other disassembly process constraints, such as the disassembly directions and tools, stability and safety.

The foundation of disassembly sequence planning is the geometric feasibility. The geometric feasible disassembly sequence represents an operation order by which components can be disassembled without interference[1]. To facilitate the geometric feasibility analysis, Bourjault[2] , De Fazio and Whitney[3,4], Homem-de-Mello and Sanderson[5] proposed a representation of the directed AND/OR graphs to create all the possible sequences. Many scholars have done a lot of researches in the field of selective disassembly sequence planning. Based on bottom-up approach, Zhang[6] proposed a new approach to obtain a selective disassembly sequence planning based on disassembly hybrid graph and particle swarm algorithm. Srinivasan H[7] used the wave propagation to study the geometric algorithm of the selective disassembly sequence generation. LI JR[8] used the hybrid graph to analyze the sequence of the target parts, and inferred the full operation of the target parts. Pan[9] proposed two kinds of selective disassembly sequence generation algorithms. Chung $\mathrm{CH}[10]$ considered batch disassembly and accessibility of the tools, and proposed an integrated method for selective disassembly. Xue and Zhang[11,12] used different algorithm to obtain the optimal sequence for a single target component based on constraints between components.

Considering geometric constraint between components and stability of disassembly process, an efficient for selective disassembly sequence planning approach for repair is proposed in this paper. 


\section{Expression of Disassembly Information}

\section{1) Precedence information}

The geometric feasible sequence means that components could be disassembled without interference by this sequence. Preference relations, which ensure the feasibility of disassembly sequence, represent the inherent and hidden geometric constraint relationships determined by the assembly structure and must be satisfied in disassembly sequence planning. References [13, 14] have a detailed description about obtaining the preference relations.

However, this kind of precedence relations is constructed based on assembly constraints, which express the geometric constraints of direct contact between components only. So a Hybrid Preference Graph (HPG) which considers the stability under gravity is constructed to represent preference relations of disassembly: $\mathrm{HPG}=\{P, E\}$, where $P$ is components set, $E$ is directed edges set. The precedence relations in HPG are expressed by directed edges. $e_{i j} \in E$ denotes the local disassembly sequence is $j \rightarrow i$. Furthermore, the hybrid precedence relations between components that have stability influence are presented by enhanced directed edge $e_{i j}$.

And then an adjacency matrix $C$ is built to denote the precedence relations among components. If directed edge $e_{i j}$ exists, $c_{i j}=1$, it means component $j$ should be disassembled before component $i$. Especially, if enhanced directed edge $e_{i j}$ exists, $c_{i j}=2$, it indicates that if component $j$ is disassembled, $i$ should be disassembled immediately to make sure that $j$ is stable during disassembly process. If directed edge $e_{i j}$ doesn't exist, $c_{i j}=0$, then the directed precedence relation between component $i$ and $j$ does not exist.

$$
c_{i j}=\left\{\begin{array}{cc}
1 & j \text { shoud be disassembled after } i \\
2 & j \text { shoud be disassembled immediatly after } i ? \\
0 & \text { there is not precedence relationship between } i \text { and } j
\end{array}\right\}
$$

2) Disassembly experience and disassembly process information

Experience is important in disassembly, but it was neglected in DSP before. Usually, there are some components that don't have precedence relations between each other, but someone does have a priori according to experience. This relation can't be deduced from geometric constraint relationships. So, an experience matrix $V=\left\{v_{i j}\right\}$ is built in this paper to express those relations that gained according to experience: if component $i$ should be disassembled immediately after $j$ is disassembled, but the directed edge $e_{i j}\left(e_{i j}\right)$ doesn't exist, $v_{i j}=1$, otherwise, $v_{i j}=0$.

\section{Selective Disassembly Sequence Planning Problem Description and Solution Method}

\section{1) Problem statements}

The definition of DSP in this paper is given below: A mechanical product $\mathrm{N}$ is composed of $\mathrm{n}$ components, $\mathrm{N}=\{1,2,3, \ldots \mathrm{n}\}$, component $p$ must be disassembled. The disassembly information includes: 1)precedence information, which is defined by HPG; 2)disassembly tool and direction of each component; and 3)other information that affects the disassembly sequence, such as stability, experience.

According to different purposes of disassembly, the optimal objectives of selective disassembly sequence planning are various. In this paper, the feasible sequences, which are efficient and stable in disassembly process, consistent with experience, are considered as the optimal or near-optimal sequence. So using the disassembly sequence $j \rightarrow i$, which means using directed edge $e_{i j}\left(e_{i j}\right)$, the optimizing criteria could be defined in four aspects as follows:(1)Stability during disassembly process: if $c_{i j}=2, S_{1}=0.1$; otherwise, $S_{1}=1$.(2)Disassembly experience: If $v_{i j}=1, S_{2}=0.1$; otherwise, $S_{2}=1$.(3)Disassembly direction change: If the direction does not change, $S_{3}=0.1$; otherwise, $S_{3}=1$.(4)Disassembly tool change: if the tool doesn't change, $S_{4}=0.1$; otherwise, $S_{4}=1$. 
So the single disassembly $i \rightarrow j$ could be evaluated: $f_{i j}(s)=\sum w_{q} s_{q}$. Where $w q(q=1,2,3,4)$ is weight coefficient. The sequence can be evaluated by following expression: $f(s)=\sum f_{i j}(s)$. The objective function could be expressed as: $f(s)=\min \sum f_{i j}(s)$.

2) Disassembled components set construction

Disassembly precedence graph is the basis of disassembly sequence planning, which can greatly reduce searching space. A part that can be disassembled means all the prior parts which constraint it are removed. These parts to be disassembled can be obtained by the following steps:

Step 1: Put target component $\mathrm{P}$ into the set $G$;

Step 2: Investigate whether $G$ is empty, if it is not, investigate all elements in the $G$. If $m$ can't be disassembled, add $m$ to set $D i$ and delete it from $G$, and then put all the prior components of $m$ into $G$. If $m$ can be disassembled, delete it from $G$. If $G$ is empty, go to step 3 .

Step 3: Output disassembly part sets Dis.

\section{Selective Disassembly Sequence Planning based on Ant Colony Algorithm}

1. Solution of assembly sequence planning

(1) Initialization. Under the guidance of the heuristic information and pheromone concentration, all the ants from the virtual node move to the next node, and traverse all the parts to be removed until the target part is removed. In order to guide ants to construct solution, a multivariate matrix $\mathrm{A}=\left[a_{i j}\right]$ is defined to express the information during disassembly process[13]. $a_{i j}=\left\{m_{i j}, \eta_{i j}, \tau_{i j}\right\}$, Where $m_{i j}$ is initialized as $c_{i j}$ and changed when component $i$ is assembled; $\eta_{i j}$ relates to the disassembly cost using $i \rightarrow j$, and it is the heuristic information of ant colony algorithm; $\tau_{i j}$, which is intercommunion medium between ants in ant colony algorithm and initialized as a constant, is the quantity of pheromone deposited on the edge $e_{i j}$.

(2) Solution searching

During solution searching, ants start from a virtual node and choose the next node to transit. When an ant is at node $i$, the next node $j$ is chosen according to the state transition rule, which is given below:

$$
p_{i j}^{k}(t)=\left\{\begin{array}{cc}
\frac{\left[\tau_{i j}(t)\right]^{\alpha}\left[\eta_{i j}(t)\right]^{\beta}}{\sum_{s \in \text { allowed }_{i}^{k}(t)}\left[\tau_{i s}(t)\right]^{\alpha}\left[\eta_{i s}(t)\right]^{\beta}} & j \in \operatorname{allowed}_{i}^{k}(t), c_{i j}=1 \\
1 & j \in \operatorname{allowed}_{i}^{k}(t), c_{i j}=2 \\
0 & \text { Else }
\end{array}\right.
$$

Where $p_{i j}{ }^{k}(t)$ is state transition probability; $q_{0}$ is a number ranging between 0 and $1 ; \tau_{i j}$ is the quantity of pheromone corresponding to the positive feedback; $\eta_{i j}=1 / f_{i j}(s)$ is the problem-dependent heuristic information corresponding to the greedy search; The exponents $\alpha$ and $\beta$ are non-negative numbers measuring the importance respectively of $\tau_{i j}$ and $\eta_{i j}$.

allowed $_{k}(i)$ represents the feasible transition candidates list when ant $k$ is at node $i$, and it is updated dynamically. According to $H P G$, component $j$ could be added into candidates list only when all of its child components are disassembled but $j$ is not disassembled. Therefore, when ant $k$ is at node $i$, the feasible transition candidates that can be chosen are updated as following steps:

1) Update $m_{i j}$ information: Set $m_{l i}=0, l=(1,2, \ldots, n)$;

2) Delete $i$ from allowed $_{k}(i)$ and Dis;

3)Add the removable nodes to $\operatorname{allowed}_{k}(i)$ : investigate all elements $n$ in the Dis, if $\sum m_{n i}$ $=O(i=1,2, \ldots, n)$, node $n$ (component $n)$ is the feasible transition candidate and can be added into allowed $_{k}(i)$.

(3) Pheromone updating 
An ant deposits a certain amount of pheromone on its route, and the pheromone volatilize at the same time. In DSP, if an ant chooses the directed edge $e_{i j}\left(e_{i j}\right)$, which means using the local disassembly sequence $i \rightarrow j$, the pheromone level of edge $e_{i j}\left(e_{i j}\right)$ is modified using the local updating rule:

$$
\tau(i, j) \leftarrow(1-\zeta) \cdot \tau(i, j)+\zeta \cdot \Delta \tau(i, j)
$$

Where $0<\gamma<1$ represents the local volatility of the pheromone. The term $\Delta \tau(i, j)=1 / f_{i j}(s)$ is the quantity of pheromone deposited by ants moving on the edge $e_{i j}\left(e_{i j}{ }^{\prime}\right)$.

Disassembly sequence evaluation should be done to find out the global-best solution and iteration-best solution once all ants have built their routes completely. The edges belonging to the global-best and iteration-best solution are reinforced with extra amounts of pheromone. And volatility of pheromone on all edges is also performed. The global updating rule is:

$$
\begin{aligned}
& \tau(i, j) \leftarrow(1-\rho) \cdot \tau(i, j)+\rho \cdot \mathrm{Q} \cdot \Delta \tau(i, j) \\
& \Delta \tau(i, j)=\left\{\begin{array}{cc}
f(L)^{-1} & e_{i j} \in L_{g b} \text { or } e_{i j} \in L_{i b} \\
0 & \text { Else }
\end{array}\right.
\end{aligned}
$$

Where $0<\rho<1$ represents the global volatility of the pheromone on the trails; $L_{g b}$ is the global-best solution and $L_{i b}$ is the iteration-best solution; $f(L)$ is disassembly sequence quality evaluated by objective function. The local pheromone updating urges the ants to find different solutions. The global pheromone updating highlights the better solution, to promote the ant to find the optimal solution. Then the iteration terminates and a new iteration starts after clearing the last iteration records until the termination condition is satisfied.

2. Framework of DSP based on ant colony algorithm

The realization of the selective disassembly sequence planning based on ant colony algorithm in this paper is given below:

Begin DSP

Parameter initialization: $C, A, \alpha, \beta, \zeta, \rho, Q, n, m$, Dis;

While (Termination conditions are not satisfied)

Do \{Empty sequence records and the candidate list, all the ants are at virtual nodes;

While (target part has been disassembled)

Do\{ While(All ants have transferred)

Do $\left\{\right.$ Generate allowed ${ }_{i}^{k}(t)$ according to the dynamic transfer strategy; calculating state transition probability and transfer; local pheromone updating; \}\}

global pheromone updating; \}

End

\section{Case Study}

The software implementation of the approach proposed above is carried out based on MATLAB. In order to verify this approach, the case in [15] is studied as a test case in this paper, as shown in Fig.3. Information of disassembly tool and direction is given in Table 1 and the HPG of the assembly is shown in Fig.2. The parameters have been chosen as follows. 


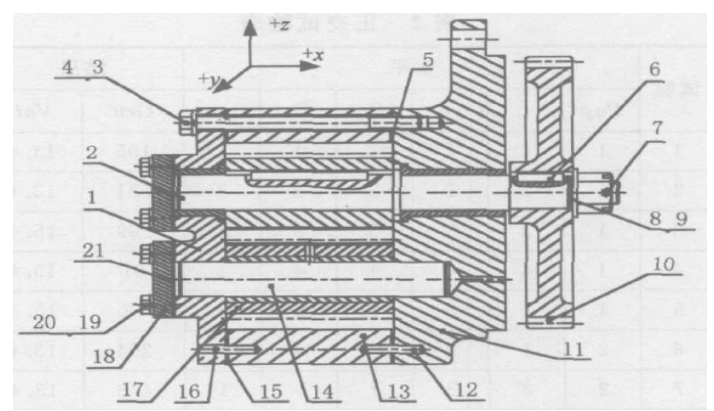

Fig. 1 Assembly of 8E150ZLC's oil pump

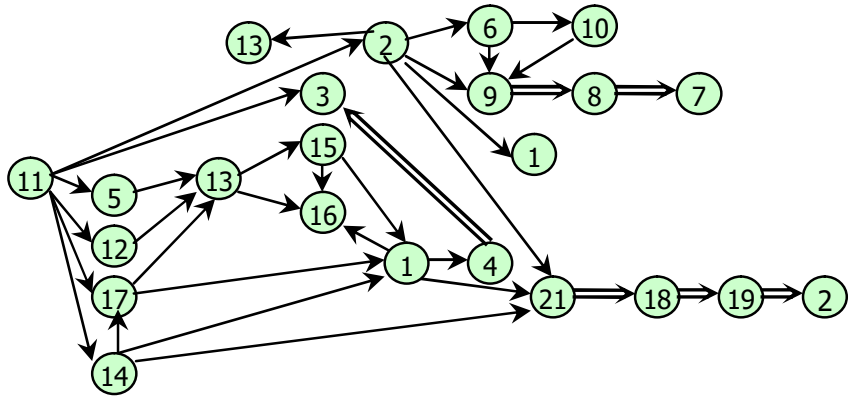

Fig. 2 HPG of 8E150ZLC's oil pump

Table 1 Information of disassembly process

\begin{tabular}{|c|c|c|c|c|c|c|c|}
\hline Number & Name & $\begin{array}{c}\text { Tool } \\
\text { number }\end{array}$ & Direction & Number & Name & $\begin{array}{c}\text { Tool } \\
\text { number }\end{array}$ & Direction \\
\hline 1 & end cover of pump & 1 & $+\mathrm{Z}$ & 12 & pin & 5 & $+\mathrm{Z}$ \\
\hline 2 & $\begin{array}{c}\text { component of driving } \\
\text { shaft }\end{array}$ & 1 & $+\mathrm{z}$ & 13 & the pump cover & 1 & $+\mathrm{Z}$ \\
\hline 3 & bolt & 2 & $+\mathrm{z}$ & 14 & stationary shaft & 1 & $+\mathrm{z}$ \\
\hline 4 & washer & 1 & $+\mathrm{Z}$ & 15 & adjusting shim & 1 & $+\mathrm{Z}$ \\
\hline 5 & adjusting shim & 1 & $+\mathrm{Z}$ & 16 & pin & 5 & $+\mathrm{Z}$ \\
\hline 6 & woodruff key & 1 & $-\mathrm{y}$ & 17 & component of driven gear & 1 & $+\mathrm{Z}$ \\
\hline 7 & pin & 3 & $-\mathrm{Z}$ & 18 & cover board & 1 & $+\mathrm{z}$ \\
\hline 8 & nut & 2 & $-\mathrm{Z}$ & 19 & washer & 1 & $+\mathrm{z}$ \\
\hline 9 & washer & 1 & $-\mathrm{Z}$ & 20 & bolt & 2 & $+\mathrm{z}$ \\
\hline 10 & gear & 1 & $-Z$ & 21 & gasket & 1 & $+\mathrm{Z}$ \\
\hline 11 & cump body & 1 & $+\mathrm{z}$ & & & & \\
\hline
\end{tabular}

The disassembly sequence planning is carried out for 10 times and the performance of the approach is shown in Fig.5. Suppose part 11 is target part. The main parameters of the algorithm used the common setting method: $m=25, \alpha=2, \beta=8, \rho=0.2, \zeta=0.1$. The disassembly planning results are shown in Fig 3 and Table 2.
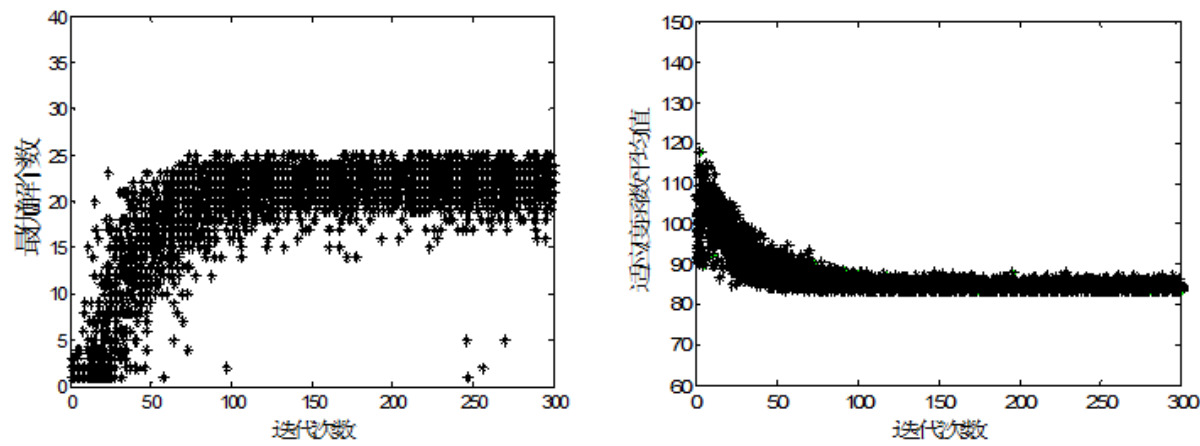

Fig 3 Disassembly planning results

Table 2 Disassembly sequence planning results

\begin{tabular}{l}
\hline Optimal or near optimal solution of disassembly sequence planning \\
\hline $7 \rightarrow 8 \rightarrow 9 \rightarrow 10 \rightarrow 6 \rightarrow 3 \rightarrow 20 \rightarrow 16 \rightarrow 4 \rightarrow 19 \rightarrow 18 \rightarrow 21 \rightarrow 1 \rightarrow 15 \rightarrow 13 \rightarrow 17 \rightarrow 14 \rightarrow 2 \rightarrow 12 \rightarrow 5 \rightarrow 11$ \\
$7 \rightarrow 8 \rightarrow 9 \rightarrow 10 \rightarrow 6 \rightarrow 20 \rightarrow 3 \rightarrow 16 \rightarrow 4 \rightarrow 19 \rightarrow 18 \rightarrow 21 \rightarrow 1 \rightarrow 15 \rightarrow 13 \rightarrow 2 \rightarrow 12 \rightarrow 17 \rightarrow 5 \rightarrow 14 \rightarrow 11$ \\
$7 \rightarrow 8 \rightarrow 9 \rightarrow 10 \rightarrow 6 \rightarrow 3 \rightarrow 20 \rightarrow 16 \rightarrow 19 \rightarrow 18 \rightarrow 4 \rightarrow 21 \rightarrow 1 \rightarrow 15 \rightarrow 13 \rightarrow 2 \rightarrow 17 \rightarrow 5 \rightarrow 14 \rightarrow 12 \rightarrow 11$ \\
\hline
\end{tabular}

Observing the results in Fig 3, it can be found that the approach is efficient. The dynamic candidates set strategy is adopted based on the precedence relations that represent the inherent and hidden geometric constraint relationships, to restrict the searching space of ant. This strategy ensures 
greater efficiency of the approach. So, the proposed approach has competitive performance for disassembly sequence generation and optimization for complex assembly. From table 2, it shows that the various solutions satisfy the geometric constraint and stability requirements. In this paper, basic part 11 is chosen as the target, and any part could be the target.

\section{Conclusion}

An approach for disassembly sequence planning based on ant colony algorithm is presented in this paper. The geometric constraints, stability and experience are taken into account in disassembly sequence planning. And the HPG is proposed to represent the geometric constraints and assembly stability. In the course of solution searching, ants start their trip from a virtual component, which is prior to all the components, and the solution space of ants is updated based on dynamic candidates set strategy. Finally, the performance of the proposed approach is verified by a cased study.

\section{References}

[1] Qiang Sua, Sheng-jie Lai and Jun Liu, "Geometric computation based assembly sequencing and evaluating in terms of assembly angle, direction, reorientation, and stability," ComputerAided Design, vol.41(2009), p.479-489.

[2] A. Bourjault, "Contribution a une Approche Methodologique de l'Assemblage Automatise," Ph.D. thesis, Department de Sciences Physiques, Universite de Franche Comte(1984).

[3] De Fazio T.L. and Whitney D.E, "Simplified Generation of All Mechanical Assembly Sequences," IEEE Trans. Robotics and Automatics, vol. 3(1987), p.640-658.

[4] Hwai-En Tseng, Wen-pai Wang, and Hsun-Yi Shih, "Using mimetic algorithms with guided local search to solve assembly sequence planning," Expert Systems with Applications, vol. 33(2007), p. 451-467.

[5] Homen de Mello L. S., and Sanderson A. C., "Representations of mechanical assembly sequences," IEEE Transactions on Robotics and Automation, vol. 7(1991), p.211-227.

[6] Zhang Xiufen, Zhang Shuyou, Yin Guodong: Object Selective Disassembly Sequence Planning for Complex Mechanical Products. Journal of Mechanical Engineering, Vol.46(2010),p.172178.

[7] SRINIVASAN H, GADH R: A geometric algorithm for single selective disassembly using the wave propagation abstraction. Computer-Aided Design, vol.30 (1998),p.603-613.

[8] LI J R, TOR S B, KHOO L P:A hybrid disassembly sequence planning approach for maintenance. Journal of Computing and Information Science in Engineering, vol.2(2002), p.2837.

[9] Pan Xiaoyong, Liu Guangfu, Liu Zhifeng: Research on Generation Method of Selective Disassembly Sequences. Machine tools and Hydraulic, vol. 4 (2003), p.42-43.

[10] CHUNG C H, PENG Q G:An integrated approach to selective disassembly sequence planning[J]. Robotics and Computer-Integrated Manufacturing, vol.21(2005),p. 475-485.

[11] Xue Junfang,Zhang Xinjian,Zhang Yingli. The Optimization of the Dismantled Order on a Target Based on the Floyd Algorithmic Method [J]. Modern Manufacturing Engineering,vol.2(2014), p.83-87.

[12] Zhang Xiaohong, Li Shiqi: Single Object Selective Disassembly Sequence Planning Based on Ant Colony Algorithm. Computer Integrated Manufacturing Systems, Vol.13(2007), p.11091114. 
[13] Xie Long, "Study of Ant-colony-optimization strategy for assembly sequence planning," Harbin: Harbin Institute of Technology,(2005).

[14] Xie Long, Fu Yi-li, and Ma Yu-lin, "Assembly sequence planning based on compound assembly graph,” Computer Integrated Manufacturing Systems. Vol.10( 2004), p.997-1002.

[15] Zhou Kaijun, and Li Dongbo: Product assembly sequences planning based on genetic simulated annealing algorithm, Computer Integrated Manufacturing Systems, vol.12(2006), p.1037-1041. 